\title{
An Internal State Variable Mapping Approach for Li- Plating Diagnosis
}

\author{
Guangxing Bai and Pingfeng Wang* \\ Department of Industrial and Manufacturing Engineering, Wichita State University \\ Wichita, KS 67208 USA
}

Li-ion battery failure becomes one of major challenges for reliable battery applications, as it could cause catastrophic consequences. Compared with capacity fading resulted from calendar effects, Li-plating induced battery failures are more difficult to identify, as they causes sudden capacity loss leaving limited time for failure diagnosis. This paper presents a new internal state variable (ISV) mapping approach to identify values of immeasurable battery ISVs considering changes of inherent parameters of battery system dynamics for Li-plating diagnosis. Employing the developed ISV mapping approach, an explicit functional relationship model between measurable battery signals and immeasurable battery ISVs can be developed. The developed model can then be used to identify ISVs from an online battery system for the occurrence identification of Li-plating. Employing multiphysics based simulation of Li-plating using COMSOL, the proposed Li-plating diagnosis approach is implemented under different conditions in the case studies to demonstrate its efficacy in diagnosis of $\mathrm{Li}$ plating onset timings.

Keywords-Li-ion battery; Li-plating; Electrochemical Model; COMSOL Multiphysics; Kriging Model

* Corresponding Author. Tel: +1 316-978-5910; E-mail: pingfeng.wang@ wichita.edu 


\section{INTRODUCTION}

As the prevalence of portable electronics and hybrid electric vehicles (HEVs), applications of lithium-ion batteries draw significant attentions due to their highlighted advantages such as high energy density, slow self-discharging rate, and no memory effect. Meanwhile, the risk of various safety critical battery failures becomes an important issue to be addressed for battery applications. Development of an advanced battery management system (BMS) could significantly mitigate the risk of potential battery failures. An advanced BMS is designed to acquire electrical data, control operational environment, monitor internal states, predict future degradation, and protect batteries from unexpected failures [1]. For a BMS, state-of-charge (SoC) and state-of-health (SoH) are two important parameters indicative of battery health condition; thus, accurately estimating them becomes a paramount task in a BMS development [2]. Tremendous works have been done to improve performance of the BMS technique [3]-[10]. Since most of batteries failure modes involve very complicated internal electrochemical reactions, accurate modeling and analysis of specific battery failure mode is extremely challenging. Thus, detecting and diagnosing those failure modes become a very important research task for broad battery applications, enabling remedial strategies and protections being implemented accordingly to avoid battery failures and prevent even hazardous system damages due to these failures. Out of many battery failure modes, Li-plating is one safety critical failure mode for lithium-ion batteries that happens at the negative electrode in the charging process [11]. Once Li-plating happens, several irreversible side reactions would occur inside the battery where numerous active lithium ions would lose their activity, which could significantly reduce the capacity of cells, leading to sudden battery capacity loss. Meanwhile, the plated lithium metal around the negative electrode could form the dendritic structure that could cause the physical damage on the separator and electrodes, leading to short 
circuits or other related critical battery failures.

Since Fuller and Doyle established the electrochemical battery model using porous electrode and concentration solution theories [12], several models have further developed to investigate the internal dynamics of lithium-ion batteries. Due to the complexity and inefficiency of the electrochemical model, several models have been developed to improve the computational efficiency without largely compromising the accuracy [13]-[18]. Recently, attentions have been paid by researchers to investigate battery failure modes using the electrochemical model. Perkins et al. [19] developed a control oriented reduced order model to determine the Li-plating by estimating several important battery parameters through simulations. Legrand et al. presented a new method to access the charging process of Li-ion battery thereby characterizing the Li-plating effect [20]. However, the existing work failed to consider the impact of changes of battery internal state variables due to aging of batteries over time. Since most related work has been built upon the foundation of battery electrochemical model, or Doyle-Fuller-Newman (DFN) model [12], that employs a system of partial differential equations (PDEs) to describe electrochemical principles in Li-ion batteries, coefficients in these PDEs are essential to estimate correct values under various battery operating conditions. While a battery system undergoes diverse variation of external circumstances in service, several physical and chemical coefficients in the PDEs could vary in a large range enough to change system behaviors. In this case, employing fixed coefficients to analyze battery system behaviors is thus inappropriate. The related time-varying coefficients in a Li-ion battery system, denoted as internal state variables (ISVs), include diffusion coefficients, reaction rate, conductivity, and so on. With regard to specific failure mode, a certain set of coefficients could be influenced to induce or mitigate the occurrence of battery failures. By identifying failure related coefficients over time, such as those key factors that activate the Li- 
plating failure mechanism, battery system behaviors could be better understood.

A few body of work has been reported in the literature that focuses on battery parameter identification by applying different approaches to given battery models. Santhanagopalan et al. [22] developed an approach that employs Levenberg-Marquardt optimization and Markov chain Monte Carlo (MCMC) method to estimate parameters in the DFN model. Speltino et al. [23] used a two-step approach to identify battery parameters, in which the cathode equilibrium potential function is first approximated from observations of open circuit voltage (OCV), and battery tests are performed to identify battery parameters of interest. Forman et al. employed a generic algorithm (GA) to identify battery parameters with the DFN model and used fisher information criterion to perform identifiability analysis [25]. In existing studies, a full DFN model has been generally used for parameter identification combined with other simulation or heuristic algorithms such as MCMC and GA. The computational burden for parameter identification is usually very high based on the complexity of numerical calculation of PDEs in the DFN model, and meanwhile the implementation of MCMC or GA also adds up a large amount of computational work, which together prevents these approaches from practical applications.

To address these challenges, this paper presents a new internal state variable (ISV) mapping approach to identify battery parameters and capture battery system failure due to Li-plating. In the developed ISV mapping approach, an artificial neural network (ANN) model is firstly constructed to model battery system responses with respect to different designed battery parameters. With the developed ANN model, weights in the NN model can be used to enhance their correlations with battery parameters in the original DFN model. Thus, a Kriging based surrogate model can then be built to map from NN weights to battery internal state variables. With the constructed Kriging model that maps the ANN weights with the battery ISVs can be further used to identify battery 
parameters in real time with online measurement data during the battery service process. After identifying battery ISVs, a new criterion to detect the occurrence of Li-plating is proposed. To demonstrate the developed ISV mapping approach for Li-plating diagnosis, the experiment is based on the COMSOL Multiphysics to simulate the Li-plating, multiphysics simulation of a battery system is implemented by using the COMSOL Multiphysics software and then the concentration information of electrodes is employed to analyze the local effects and the onset timing of Li-plating.

The rest of the paper is organized as follows. Section 2 introduces the related work on Li-ion battery electrochemical model and the ANN and Kriging models used for the development of ISV mapping approach. The section 3 details the developed ISV mapping approach. The section 4 presents the mechanisms of Li-plating and the new approach for the detection of Li-plating. The section 5 shows the experiments implemented by COMSOL and the results that illustrate the Liplating diagnosis using the developed approach.

\section{RELATED WORK}

In this section, the previous valuable work will be introduced, which includes the description of the pseudo 2D battery model in subsection 2.1. The artificial neural network model and the Kriging model used for the developed ISV mapping approach are briefly introduced in subsections 2.2 and 2.3 , respectively.

\subsection{The Doyle-Fuller-Newman (DFN) Model}

The DFN model, or pseudo two dimensional (P2D) model, was developed from the battery 
electrochemical model with the ohmic porous electrode theory and Bulter-Volmer kinetics by Fuller et al. [12]. Based on the battery electrochemical kinetics, the pseudo 2D model can describe the internal variables such as concentration or potential in both solid phase and solution phase. The P2D model consists of three regions: negative electrode, separator, and positive electrode. In each electrode, there are two phases which are the solid phase consisting of porous electrode particles and the solution phase being full of electrolyte. At regularly charging/discharging processes, the current will force the Li-ions to extract from particles of one electrode, move through the electrolyte, and intercalate into particles of the other electrode. The P2D model describes this behavior of Li-ions which follows the diffusion kinetics [21].

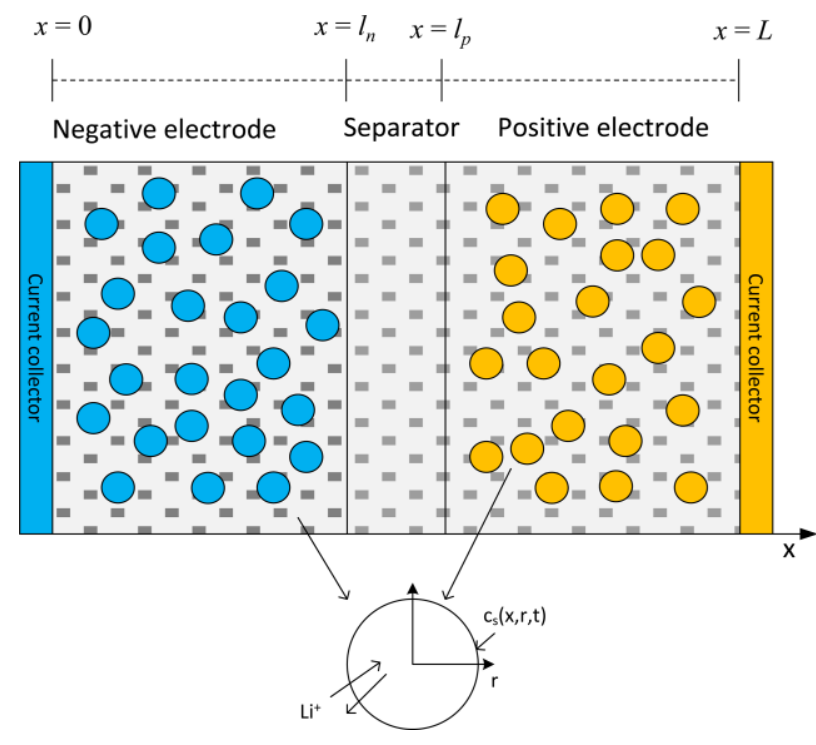

Figure 1. The 2D structure of a Li-ion battery

As shown in Fig. 1, a generic P2D model structure is used to study the battery internal states. This model only considers $\mathrm{Li}^{+}$diffusion dynamics in electrolyte along the horizontal $\mathrm{x}$-axis and $\mathrm{Li}^{+}$exaction/intercalation behaviors in spherical particles along the radius r-axis. The partial differential equations (PDEs) are employed to solve for the electrolyte concentration, electrolyte 
potential, solid phase concentration, and solid phase potential along the different locations in $\mathrm{x}, \mathrm{r}$ axis. The detailed equations of the P2D model is presented on Appendix A.

\subsection{Review of the Artificial Neural Network Model}

Artificial neural networks (ANN) have been designed for various applications, such as pattern recognition, prediction, optimization, function approximation, and control. In this paper, one of the most commonly used ANN, namely a multilayer perceptron (MLP), is employed to approximate the system response for a dynamic battery system. In a MLP, several neuron nodes are used to compose three types of network layers, with generally one input layer, one or multiple hidden layers, and one output layer. The information is propagated directly from the input layer through hidden layers to the output layer. The information propagation process is realized by using the activation function, which transforms the activation level of a neuron into an output signal. There are a number of common activation functions in use with ANN, and out of which the sigmoid function is most widely used. A general ANN model is showed on Figure 2.

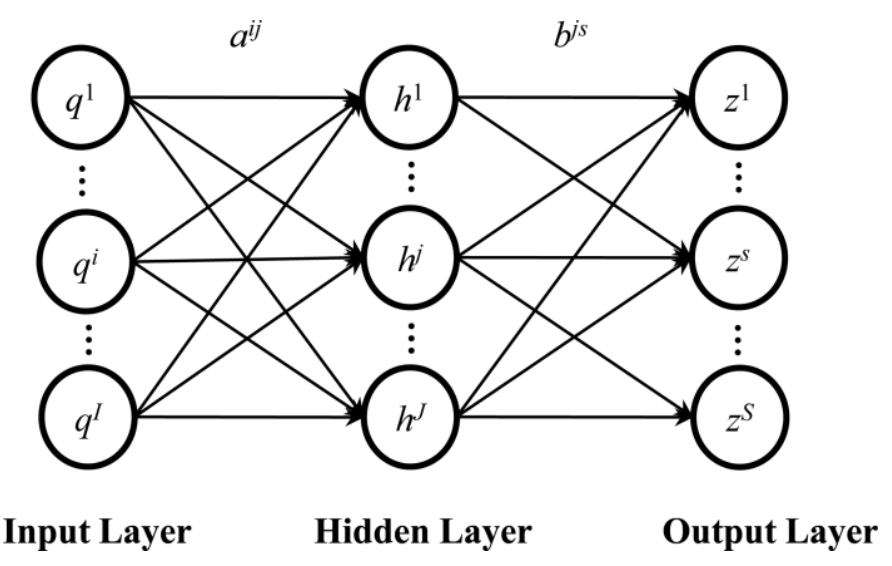

Figure 2: A general neural network model 
In this study, an ANN is constructed to intelligently learn response of the P2D model with multiple coefficients, then a Kriging model could find the relationship between ANN weights and P2D model coefficients.

\subsection{Review of the Kriging-based Surrogate Model}

This subsection introduces the Kriging technique, which is a probabilistic method for interpolating values modeled by a Gaussian process based on observed measurements. Based on various stationary properties of global means in Kriging model, different methods were developed to apply practical situations of various degrees of stationarity assumed. In this paper, the ordinary Kriging as one of classical Kriging methods is employed to address the special ISV mapping problem.

The Kriging model is usually presented as

$$
y(\mathbf{x})=f(\mathbf{x})+S(\mathbf{x})
$$

where $\mathbf{x}$ is an $n$-dimensional vector for $n$ design variables, $y(\mathbf{x})$ is an unknown function that the Kriging model tends to express, $f(\mathbf{x})$ is a global mean function that can provide several options such as a constant term or a polynomial function, and $S(\mathbf{x})$ is the realization of a stochastic process with zero mean and variance $\sigma^{2}$, which represents the local deviation from the global mean. In this paper, a constant $\mu$ is used for the global mean function $f(\mathbf{x})$.

The correlation between $S\left(\mathbf{x}_{i}\right)$ and $S\left(\mathbf{x}_{j}\right)$ is related to the distance between two samples $\mathbf{x}_{i}$ and $\mathbf{x}_{j}$. It is represented as 


$$
\operatorname{Corr}\left(\mathrm{S}\left(\mathbf{x}_{i}\right), \mathrm{S}\left(\mathbf{x}_{j}\right)\right)=\sigma^{2} R\left(\mathbf{x}_{i}, \mathbf{x}_{j}\right)
$$

where $R\left(\mathbf{x}_{i}, \mathbf{x}_{j}\right)$ is an $n \times n$ correlation matrix, and the distance between $\mathbf{x}_{i}$ and $\mathbf{x}_{j}$ is not the Euclidean distance that has the same weights for all design variables. The distance with additional weighted values can be expressed as

$$
d\left(\mathrm{x}_{i}, \mathrm{x}_{j}\right)=\sum_{k=1}^{n} \alpha_{k}\left|\mathrm{x}_{i}^{k}-\mathrm{x}_{j}^{k}\right|^{2}
$$

where $\alpha_{k}$ are the undetermined weights used to match the model, and $\mathbf{x}_{i}^{k}$ and $\mathbf{x}_{j}^{k}$ are the $\mathrm{k}^{\text {th }}$ design variables. Given the distance of two samples, the $(i, j)$ entry of correlation matrix $R$ can be written as

$$
R\left(\mathbf{x}_{i}, \mathbf{x}_{j}\right)=\exp \left(-d\left(\mathrm{x}_{i}, \mathrm{x}_{j}\right)\right)
$$

For a new interpolated sample $x_{n e w}$, the prediction of the Kriging model is given by

$$
y\left(\mathrm{x}_{\text {new }}\right)=\mu^{\prime}+\mathbf{r}^{\mathrm{T}}\left(\mathrm{x}_{\text {new }}\right) \mathrm{R}^{-1}\left(\mathbf{y}-\mathbf{1} \mu^{\prime}\right)
$$

where $\mathbf{r}\left(x_{\text {new }}\right)$ is a correlation vector with the $i^{\text {th }}$ element is

$$
r_{i}\left(\mathrm{x}_{\text {new }}\right)=\operatorname{Corr}\left(\mathrm{S}\left(\mathrm{x}_{\text {new }}\right), \mathrm{S}\left(\mathrm{x}_{i}\right)\right)
$$

and the mean estimate $\mu^{\prime}$ is

$$
\mu^{\prime}=\left(\mathbf{1}^{\mathrm{T}} \mathbf{R}^{-1} \mathbf{1}\right)^{-1} \mathbf{1}^{\mathrm{T}} \mathbf{R}^{-1} \mathbf{y}
$$

Also, the estimate of the variance, $\sigma^{\prime}$ is defined by

$$
\sigma^{\prime}=\frac{\left(\mathbf{y}-\mathbf{1} \mu^{\prime}\right)^{\mathrm{T}} \mathbf{R}^{-1}\left(\mathbf{y}-\mathbf{1} \mu^{\prime}\right)}{n}
$$

Among all equations, the undetermined parameter in Kriging model is $\boldsymbol{\alpha}$ in Eq. (3). In order to estimate this parameter, the maximum likelihood estimation (MLE) method is employed to address this problem. The likelihood function is expressed as 


$$
\text { Likelihood }\left(\mu^{\prime}, \sigma^{\prime}, \boldsymbol{\alpha}\right)=-\frac{n}{2} \ln (2 \pi)-\frac{n}{2} \ln \left(\sigma^{\prime 2}\right)-\frac{1}{2} \ln (|\mathbf{R}|)-\frac{1}{2 \sigma^{\prime 2}}\left(\mathbf{y}-\mathbf{1} \mu^{\prime}\right)^{\mathrm{T}} \mathbf{R}^{-1}\left(\mathbf{y}-\mathbf{1} \mu^{\prime}\right)
$$

In order to maximize Eq. (9), several numerical methods such as Newton-Gauss method are selected to approximate model parameter $\boldsymbol{\alpha}$. For given $\boldsymbol{\alpha}, \mu^{\prime}$ and $\sigma^{\prime}$ are able to be updated accordingly. The calculated routine then repeats calculating these three coefficients until likelihood function converges to a stable maximum value.

\section{THE DEVELOPED ISV MAPPING APPROACH}

This section presents the developed ISV mapping approach. Subsection 3.1 briefly presents the battery parameter estimation problem. After simplifying the Li-ion battery P2D model in subsection 3.2, the ISV mapping approach to estimate PDE coefficients is then detailed in subsection 3.3. Subsection 3.4 shows the flowchart that concludes the procedures of the developed approach.

\subsection{Identification of Battery Model Parameters}

As previously mentioned in Section 1, system inherent parameters must be identified and confirmed so that system internal states can be estimated during battery system dynamical processes. However, determining the coefficients in PDEs is a very challenging problem especially in a complex PDE form or system of PDEs [22]-[24]. Meanwhile, existing studies generally employ the full or reduced-order P2D models, and rely on numerical techniques to iteratively estimate required parameter values. To apply existing methods for estimations of PDE coefficients, computational burden is generally extremely high for even a single battery parameter. Mathematically, the presented problem for a PDE can be expressed as 


$$
F\left(\mathbf{x}, \mathrm{t}, \mathrm{u}, \frac{\partial u}{\partial x_{1}}, \ldots, \frac{\partial u}{\partial t} ; \boldsymbol{\theta}\right)=0
$$

where $F$ is a linear function of $u$ and its derivatives, $\mathbf{x}=\left(x_{1}, \ldots x_{p}\right)^{\mathrm{T}}$ is a p-dimensional argument, $\boldsymbol{\theta}$ is the parameter vector that relates to primary interest in this paper.

The present problem for Li-ion battery electrochemical model is more complex than Eq. (10). First, the P2D model includes several PDEs from Eq. (A.1) to Eq. (A.16) to construct a system of PDEs in order to describe the entire Li-ion battery system. Second, the variable $\mathbf{u}$ in Eq. (10) is unobservable in system of PDEs for Li-ion battery, which increases difficulty with using observations to estimate PDE parameters. For the sake of addressing these challenges, an ISV mapping approach is proposed. In the new approach, a reduced order model is developed for P2D model to reveal dependency between observed voltage and variables in the battery model, and a surrogate model is applied for reduce the computational complexity of the system of PDEs.

\subsection{Reduced Order Model for Li-ion Battery}

This subsection presents the reduced order model for Li-ion battery P2D model. Recalling the Eq. (A.3), the output voltage equals to the difference between potentials at two boundaries $x=0$ and $x=L$. Since we don't have to consider the $x$ variables for calculating output voltage, the existing PDE can be reduced to an ordinary differential equation (ODE) for electrode potential as follows:

$$
\mathrm{L} \Phi(x, t)=f(x, t, I(t), \boldsymbol{\theta}) \rightarrow \frac{\mathrm{d} \Phi_{x=0, L}(t)}{\mathrm{d} t}=f(t, I(t), \boldsymbol{\theta})
$$

Thus, Eq. (11) simplifies the variable $\Phi$ to be a variable only related to time. By discretizing this 
equation, we can obtain

$$
\Phi_{x=L, k}-\Phi_{x=L, k-1}=\Delta t \times f\left(I_{k}, \boldsymbol{\theta}\right)
$$

Finally, the voltage at time index $k$ can be summarized as

$$
V_{k}=G\left(I_{k}, V_{k-1}, \boldsymbol{\theta}\right)
$$

The Eq. (13) implies that the output voltage at time point $k$ only depends on the input current at time $k$, voltage at last time point $k-1$, and the related coefficients in PDEs. The Eq. (13) can significantly reduce the complexity of the original system of PDEs and easily generalize the relationship between system input and output. Base on Eq. (13), it has a great convenience to employ a surrogate model to replace the reduced model and estimate system response with required accuracy.

\subsection{Battery ISV Mapping}

This subsection presents the proposed the methodology of the developed approach. To realize the time-varying parameters, the diffusion coefficients, in the governing PDEs, a two-stage method is designed to solve this problem.

In the first stage, a surrogate model is constructed to approximate PDE system responses. A surrogate model has two advantages compared to the original full PDE model: first, under an accepted accurate level, a surrogate model could largely reduce the computational complexity;

second, a surrogate model provides explicit weights in model equations, which could be utilized to map time-varying coefficients in a PDE model.

In the developed approach, a neural network (NN) model is selected from surrogate models. The NN model can be written as 


$$
y(x)=\sum_{i=1}^{I} a_{i} \varphi\left(\sum_{j=1}^{J} w_{i j} x_{j}+b_{j}\right), \text { and } \mathbf{W}=\left[a_{i}, w_{i j}, b_{j}\right]^{\mathbf{T}}
$$

where $a, w$, and $b$ are all weights inside the NN model, $x$ is the input of NN model, $i$ and $j$ represent the number of hidden nodes and input nodes, respectively. $\varphi$ is the sigmoid function that can be expressed as

$$
\varphi(\xi)=\frac{1}{1+e^{-\xi}}
$$

In order to surrogate the reduced battery model, the input of NN model is assigned to be $I_{k}$ and $V_{k}$ ${ }_{1}$; the output of NN model is $V_{k}$.

To fit the battery model, the Levenberg-Marquardt algorithm is used to optimize NN weights to ensure the validation of $\mathrm{NN}$ model. The optimization is expressed as

$$
\underset{\mathbf{W}_{\text {cycle }}}{\operatorname{Minimize}}: \sum_{k}\left(V_{k, \text { cycle }}-y\left(\mathbf{W}_{\text {cycle }}\right)\right)^{2}
$$

where cycle represents the cycle index, $k$ is the time index in a specific cycle, $y$ represents the estimates of $\mathrm{NN}$ model, and $V$ is the true system output.

Apparently, the trained weights $\mathbf{W}$ in the NN model have a strong correlation with coefficients $\boldsymbol{\theta}$ since both of them are the only parameters in their respective models. Therefore, building an accurate mapping from $\mathbf{W}$ to $\boldsymbol{\theta}$ would assist us to determine the values of PDE coefficients. The mapping can be written as

$$
g: \mathbf{W} \rightarrow \boldsymbol{\theta}
$$

In this mapping, the dimension of input is regulated by the number of hidden nodes in the predefined NN model, and the dimension of output is determined by the number of coefficients in 
PDEs. The mapping solution in this paper is Kriging model that has the powerful estimation on interpolation problems.

\subsection{Flowchart of the ISV Mapping Approach}

In this subsection, the procedure of the developed approach, as illustrated by the Fig. 3, is presented. According to the proposed procedure, the whole approach is divided into offline and online stages. The basic idea is to utilize the mapping relations between NN weights and diffusion coefficients revealed by Kriging model to estimate the current diffusion coefficients in the testing battery with experimental data. In the offline stage, the testing battery characteristics, such as materials in electrodes and electrolyte, geometry of the battery structure, and several other fixed parameters, have to be designed in a COMSOL multiphysics model, then this model is able to be executed the simulated battery cycling experiments that could easily provide the system response under different designed current and diffusion coefficients. After obtaining system behavior with several I-V curves, a NN model is employed to approximate the I-V relations instead of using a full PDE model. As mentioned previously, a Levenberg Marquardt algorithm is used to optimize the NN model until the required accurate level is reached. Due to the direct relations between weights from the NN model and diffusion coefficients from the aimed PDE model, a Kriging model is employed here to approximate the mapping relations from weights $\mathbf{W}$ to coefficients $\boldsymbol{\theta}$.

In the online stage, the testing battery has to be set up to go through a charging and discharging experiment to obtain the real experimental data. The battery experiment firstly performs a discharging profile until the voltage drops under $3.2 \mathrm{~V}$ that is the stop voltage for discharge. The battery could be considered as deeply discharged in this case. Then the discharged battery is applied on a constant charging current, and at the same time, the battery tester is 
recording the measurement signals: current and voltage. In the same procedure, the measured I-V curve is also plugged into the NN model to get trained until the error level is met. Obtaining the weights $\mathbf{W}$ ' from new trained NN, we plug the $\mathbf{W}$ ' into the designed Kriging model and get the prediction of diffusion coefficients $\boldsymbol{\theta}$ '. Since the diffusion coefficients $\boldsymbol{\theta}^{\boldsymbol{\prime}}$ represent the battery current states that could affect the Li-plating occurrence, the Li-plating could be predicted according to the given diffusion coefficients.

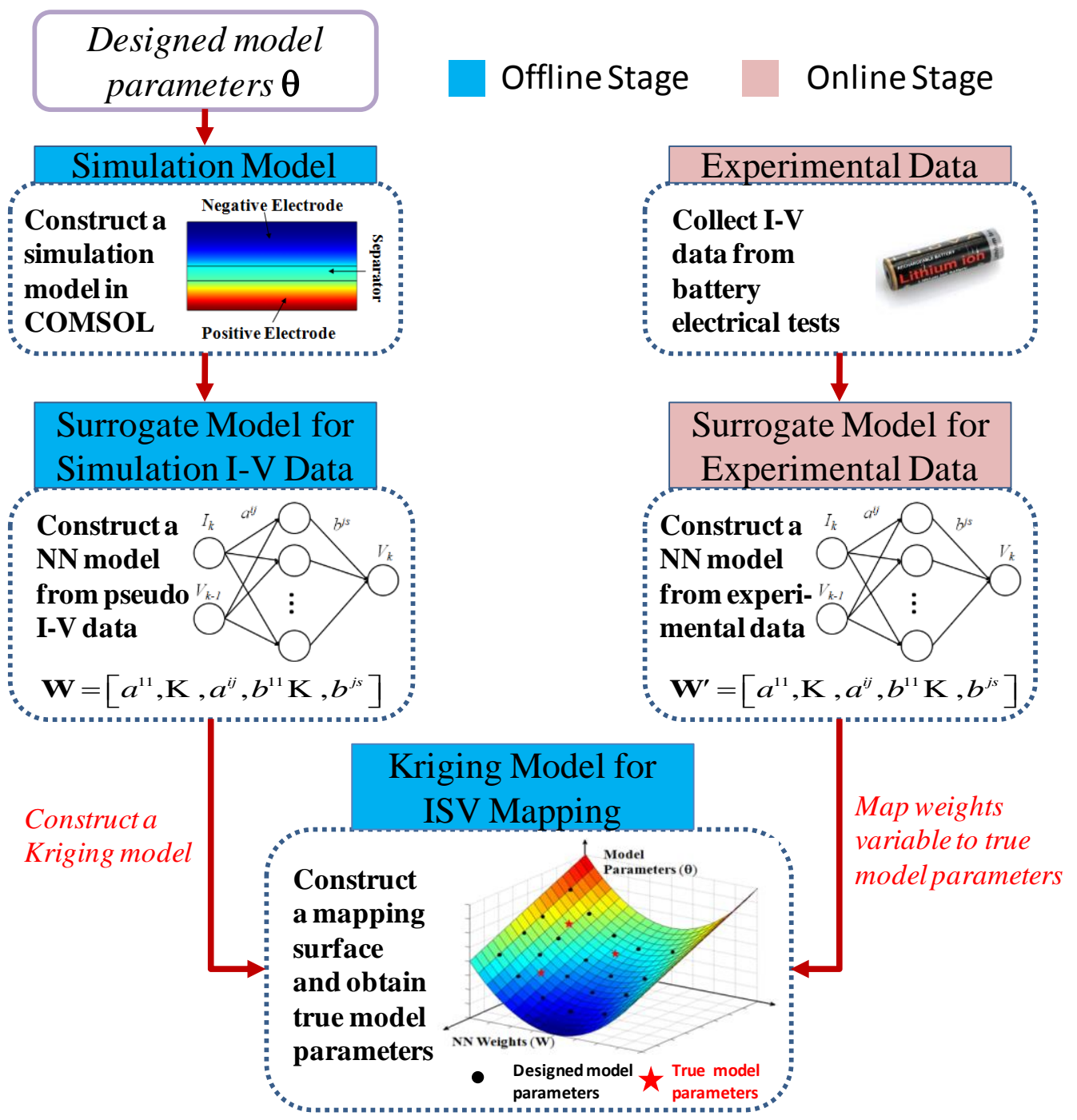

Figure 3. Procedure of the developed ISV mapping approach 


\section{LI-PLATING MECHANISM}

In this section, the Li-plating mechanism is briefly reviewed and the developed Li-plating occurrence model for diagnosis is presented. In the following, Subsection 4.1 introduces the Liplating mechanism and Subsection 4.2 presents essential assumptions and the proposed Li-plating occurrence model.

\subsection{Electrochemical Explanations for Li-plating Mechanism}

Under extremely operating conditions that make reduction reactions occur easily, metallic lithium could be deposited (plated) on the surface of the negative electrode instead of intercalating into it. Li-plating phenomenon generally occurs in the charging process under two specific conditions: charging at high current or low temperature.

When Li-ion batteries are charging at high current, the active Li-ion will extract from the positive electrode, move across the separator, and intercalate into the negative electrode. However, the high current makes the extraction rate larger than the intercalation rate, and then side reactions will occur and turn Li-ions to metallic lithium on the surface of negative electrodes

particles. Similarly, the low temperature will slow down the intercalation rate on the surface of negative electrode and result in the Li-plating on the negative electrode. These side reactions when Li-plating occurs are concluded as follows:

$$
\begin{gathered}
x \mathrm{Li}^{+}+\mathrm{Li}_{\delta} \mathrm{C}_{6}+x e^{-} \rightarrow \mathrm{Li}_{\delta+x} \mathrm{C}_{6} \\
(1-x) L i^{+}+(1-x) e^{-} \rightarrow(1-x) L i \downarrow
\end{gathered}
$$

The reaction (17) is the regular insertion reaction in which $x \mathrm{Li}^{+}$( $x$ represents the fraction of 
charge carriers) insert into the graphite structure formed by six carbon atoms $C_{6}$. The reaction (18) is the Li-plating side reaction which causes that $(1-x) L i^{+}$forms metal lithium and is plated on the surface of negative electrodes.

Since the metallic lithium has very active properties, it will continue having other side reactions with substances in electrolyte, which are shown as follows:

$$
R+L i \downarrow \rightarrow L i-R
$$

where $\mathrm{R}$ represents the carbonate solvent electrolyte. This side reaction indicates that metallic lithium could be rapidly oxidized by the substances of electrolyte and form an additional SEI [19]. The new SEI layer could stop the further side reactions between plated lithium metal and other oxidizers. As a consequence, the new-formed plated lithium metal has been protected gradually and will form a dendritic form that would cause more hazardous damages.

\subsection{Li-plating Occurrence Model for Diagnosis}

As we described in the subsection $\mathrm{A}$, the Li-plating occurs when the extraction rate of $\mathrm{Li}^{+}$ from positive electrodes exceeds the intercalation rate of $\mathrm{Li}^{+}$into negative electrodes. To create the Li-plating model, several additional assumptions should be claimed: (1) the reaction (19) as the only side reaction occurs in the charging process. The other side reactions have to be ignored, and (2) the $\mathrm{Li}^{+}$concentration gradient on the surface of electrodes approximates to the extraction or intercalation rate, because the Li-plating only relates to the variation of $\mathrm{Li}^{+}$concentration on the surface of electrode particles. Besides, in order to compare two rates in local areas, the average concentration gradient could be computed in a specific area. Based on this fact, we defined two equations to demonstrate the extraction/intercalation rate as follows: 


$$
\begin{aligned}
& R_{e x}=\frac{\partial \bar{c}_{s, s u r f, p}}{\partial t} \\
& R_{i n}=\frac{\partial \bar{c}_{s, s u r f, n}}{\partial t}
\end{aligned}
$$

where $R_{e x}$ and $R_{i n}$ represent the extraction rate and intercalation rate, respectively, $c_{s, s u r f, p}$ and $c_{s, s u r f, n}$ represent the solid phase concentration on the surface of different electrodes particles, which can be obtained by $c_{s, \text { surf }}=\left.c_{s}\right|_{r=R p}$ for both electrodes according to Eq. (A.10). After calculating Eqs. (20) and (21) at different locations and time points, the extraction and intercalation rates could be obtained. According to the mechanism of the Li-plating, the Li-plating occurs only when the following equation is satisfied:

$$
\left|R_{e x}\right|>\left|R_{\text {in }}\right|
$$

When Eq. (22) is satisfied at a specific location or time point, the Li-plating could be considered to happen.

\section{LI-PLATING DIAGNOSIS EMPLOYING THE ISV MAPPING APPROACH}

This section presents the results of Li-plating using COMSOL Multiphysics. The subsection 5.1 introduces a case study of Li-ion battery 2D model to implement the developed approach, and the subsection 5.2 presents a case study of the battery $2 \mathrm{D}$ model to demonstrate the local Liplating effect and diagnosis using the developed ISV mapping approach.

\subsection{Battery 2D Model Case}

In this subsection, the weights-mapping approach is implemented for a Li-ion battery 2D case. The results discuss the local effects of Li-plating happening due to impact of geometry of a battery. 
The battery 2D model implemented in COMSOL Multiphysics V4.4 is a double dimension model based on electrochemical principles of a Li-ion battery. With the same materials, this model is built in three regions: the positive electrode (the pink area), the negative electrode (the grey area), and the separator (the green area). There are two current collectors covered around the two electrodes. For the experimental purpose, the current collector of the positive electrode has been cut over than half areas to enhance the Li-plating effects on local areas. Thus, the battery model has been divided into two parts according to the partially covered current collector. The reason of this unbalanced design is because Li-plating could occur more rapidly in the local area than the rest test area, thereby saving a large amount of computational efforts to generate the Liplating phenomenon and data. The geometry of Li-ion battery 2D model is shown in Figure 4.

In this case study, the designed diffusion coefficients in different electrodes vary both from $1 \mathrm{e}-12$ to $1 \mathrm{e}-15 \mathrm{~m}^{2} / \mathrm{s}$ according to surveys with different measurement techniques in current literature [27]. For each diffusion coefficient, the PDE solver is designed to uniformly sweep the coefficient range as 20 steps, which implies that the total number of parameters combinations is $20 * 20=400$ groups. Using all of parameters combinations, 400 charging cycles are able to be generated to implement the developed approach. By applying the weights-mapping approach, a $\mathrm{NN}$ model is used to surrogate the designed battery model and 400 weights vectors are trained corresponding to the related parameters combinations. Then, we randomly choose 50 groups of parameters combinations to validate the developed approach. 


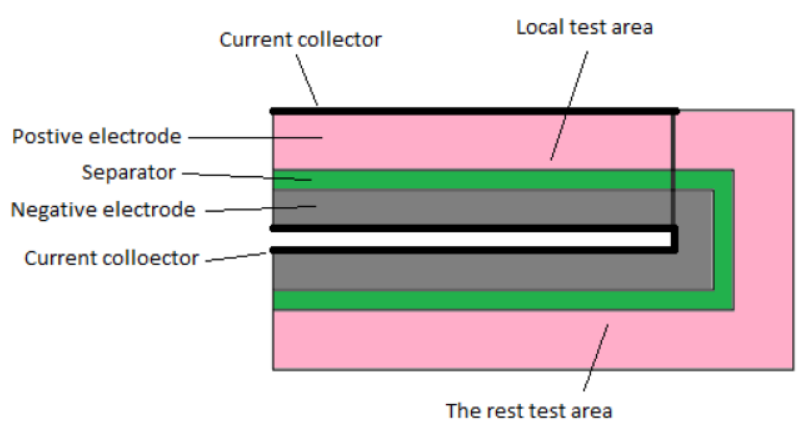

Figure 4. The Li-ion battery 2D geometry in COMSOL

\subsection{Li-plating Diagnosis Using ISV Mapping}

By applying 50 groups of testing parameters combinations, the developed approach is implemented to approximate the true PDE parameters. Figures 5(a) and 5(b) show the estimations of the diffusion coefficients at two electrodes from the Li-ion battery 2D model.

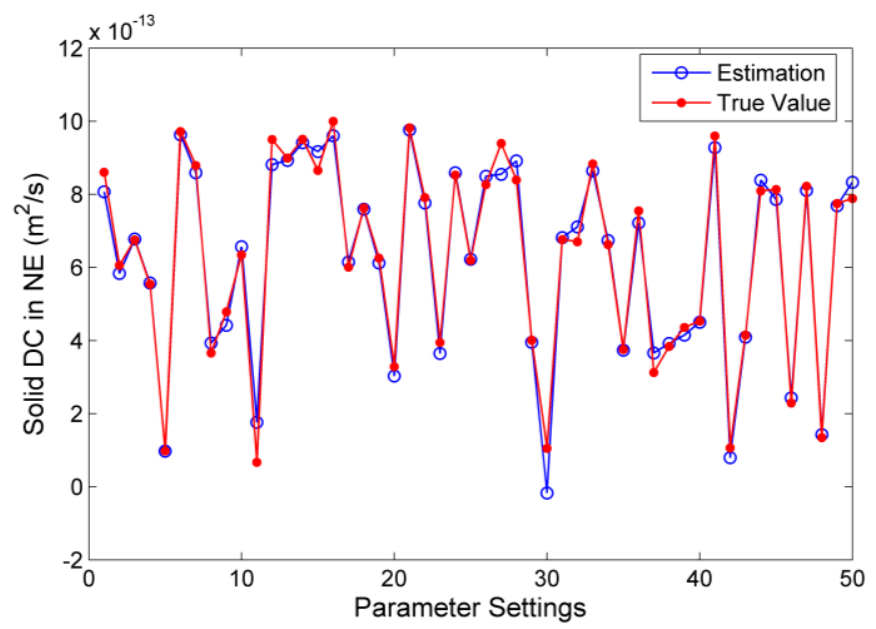

(a) Estimation of DC in negative electrode (NE) 


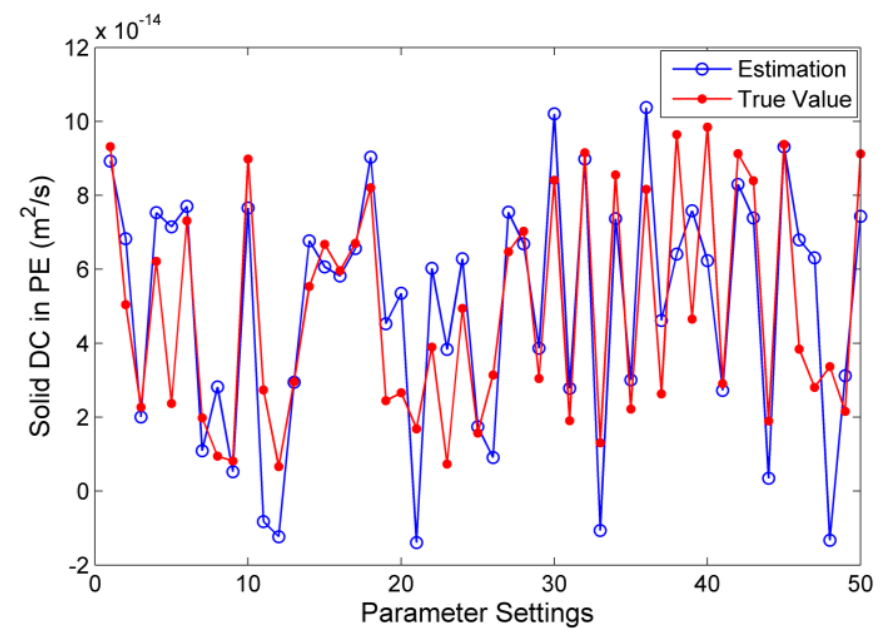

(b) Estimation of DC in positive electrode (PE)

Figure 5. Estimation of diffusion coefficients (DC) in electrodes

Table 1. Accuracy of the ISV mapping approach

\begin{tabular}{ccc}
\hline \hline Diffusion Coefficient Estimation & MSE & Relative Error (RE) \\
\hline At negative electrode & $1.3471 \mathrm{e}-27$ & $1.6414 \%$ \\
At positive electrode & $4.0126 \mathrm{e}-28$ & $4.2338 \%$ \\
\hline \hline
\end{tabular}

The results, as shown in Fig. 5(a) and 5(b), and the Table 1, indicate that the developed approach is able to achieve a high accuracy of approximation. It is observed that MSE or RE of diffusion coefficient at the positive electrode is larger than the one at the negative electrode. This fact is attributed that the ANN output, namely the voltage, has less sensitivity with diffusion 
coefficient at the positive electrode. Thus, the weights of ANN has weaker link to the diffusion coefficient at the positive electrode compared to the one at the positive electrode. The equations of mean square error (MSE) and relative error (RE) are expressed as:

$$
\begin{gathered}
M S E=\frac{1}{N} \sum_{i=1}^{N}\left(Y_{i}^{a p p r o x}-Y_{i}^{o b}\right)^{2} \\
R E=100 \% \times \frac{1}{N} \sum_{i=1}^{N}\left|1-\frac{Y_{i}^{a p p r o x}}{Y_{i}^{o b}}\right|
\end{gathered}
$$

where $N$ is the total number of approximations, $Y_{i}^{a p p r o x}$ and $Y_{i}^{o b}$ are the approximation and true value, respectively.

After obtaining the approximation of diffusion coefficients, the PDE solver could obtain the concentration information in different electrodes. For easily occurrence of Li-plating, the related parameters should be adjusted to proper values. The initial SoC has been set to $90 \%$, which means the battery is close to fully charged. Then the battery charging simulation is undergoing with different diffusion coefficient combinations. Since diffusion coefficients could significantly impact the Li-ion concentration performance in different electrodes, randomly coupled coefficients are selected to indicate the Li-plating occurrence in various conditions. Figure 6 shows the concentration gradient performance with different combinations of parameters. 


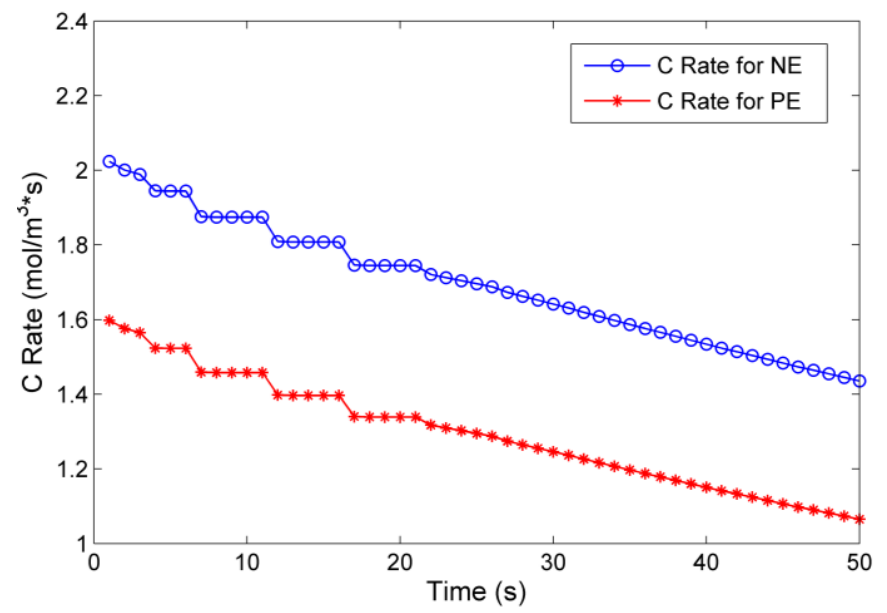

(a) Concentration gradient with $\mathrm{D}_{\mathrm{n}}=2.013 \mathrm{e}-13$ and $\mathrm{D}_{\mathrm{p}}=1.121 \mathrm{e}-13$

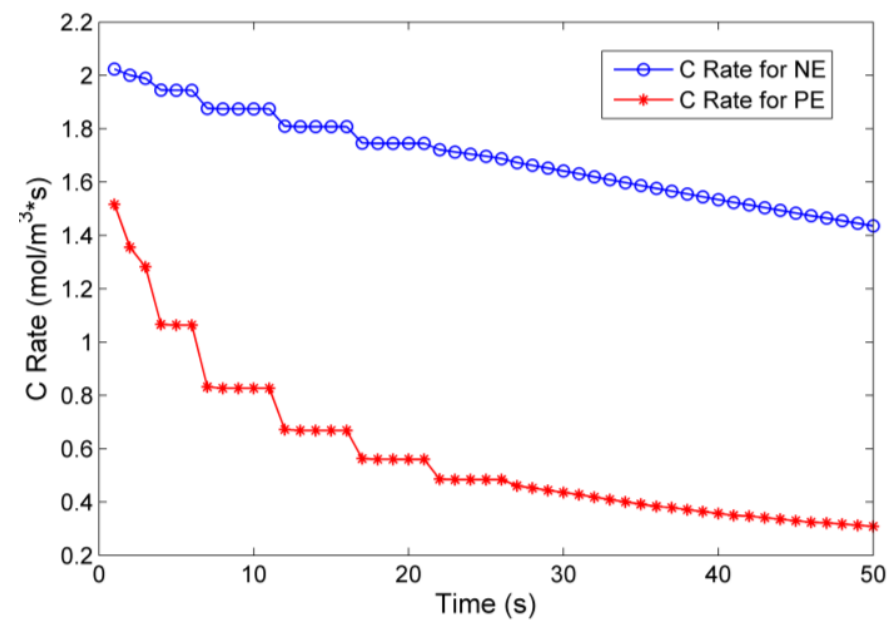

(b) Concentration gradient with $\mathrm{D}_{\mathrm{n}}=2.021 \mathrm{e}-13$ and $\mathrm{D}_{\mathrm{p}}=5.012 \mathrm{e}-14$ 


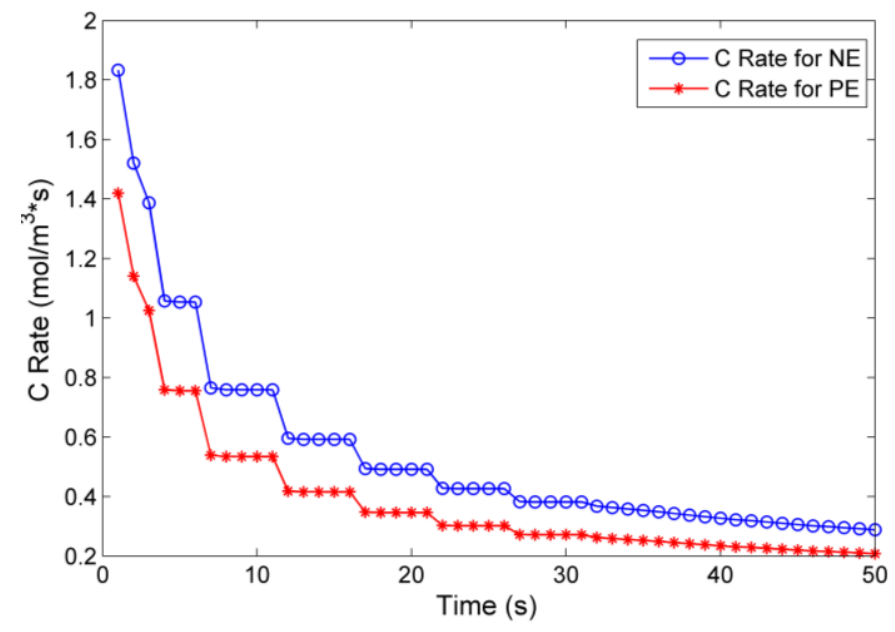

(c) Concentration gradient with $\mathrm{D}_{\mathrm{n}}=1.211 \mathrm{e}-14$ and $\mathrm{D}_{\mathrm{p}}=4.986 \mathrm{e}-15$

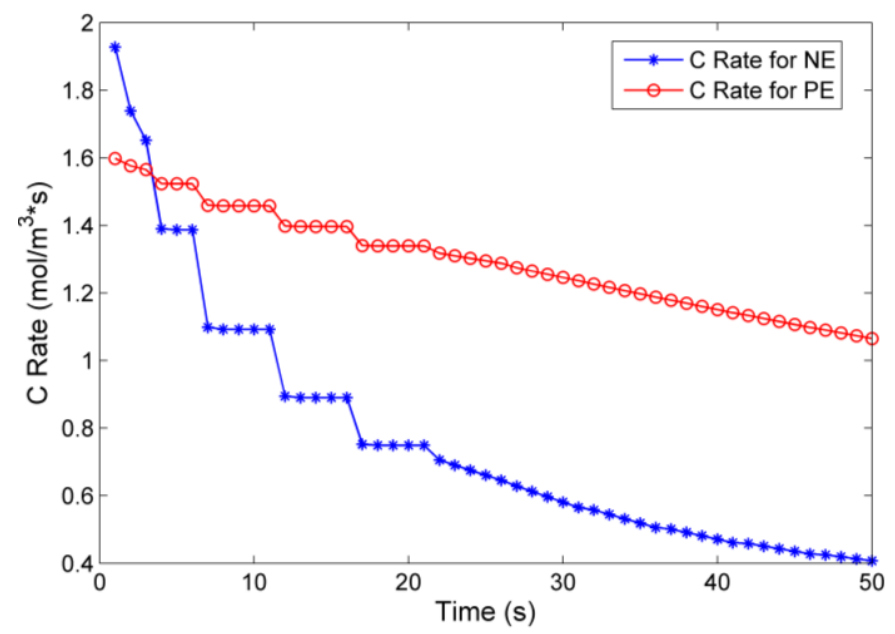

(d) Concentration gradient with $\mathrm{D}_{\mathrm{n}}=1.122 \mathrm{e}-14$ and $\mathrm{D}_{\mathrm{p}}=5.124 \mathrm{e}-14$

Figure 6. Concentration gradient with different combinations of parameters

The blue curve and red curve represent Li-ion concentration gradient for negative and positive electrodes, respectively. In Fig. 6(a), the specific diffusion coefficients make concentration gradients of both electrodes to parallel to each other, and the value of positive electrode is much smaller than the one of negative electrode. In this case, Li-plating would not happen in the entire 
time scale. In Fig. 6(b), the concentration gradient of positive electrode diverges to the other one, which indicates that Li-plating won't occur most unlikely in the future. In Fig. 6(c), the concentration gradients of both electrodes are close and converging to each other, which indicates that Li-plating could happen in the future when one curve intersects another. In the circumstance as shown in Fig. 6(d), the blue curve intersects with the red curve at time $t=4 \mathrm{~s}$, indicating that the concentration gradient of positive electrode exceeds the one of negative electrode. In this case of coupled coefficients, Li-plating would occur after the intersection and be diagnosed by the proposed approach.

\section{CONCLUSION}

In this paper, Li-plating failure identification is investigated based on capturing battery parameters dynamics. To capture system dynamics in DFN model, a new internal variable (ISV) mapping approach is developed to identify battery parameters in a system of PDEs. Multiphysics-based simulation of battery system dynamics with designed battery parameters are implemented within the COMSOL multiphysics simulation environment. With the simulation data, an artificial neural network (ANN) model is constructed to model battery system output voltage instead of DFN models, and then the links between weights in the ANN model and parameters in the DFN model is evaluated by employing a Kriging based surrogate model. Using the Kriging model, the real battery parameters can be identified from experimental data. The Liplating mechanism has been discussed and a new determining approach for Li-plating occurrence is proposed to analyze the local effects and the onset timing of Li-plating under the high current conditions by using the developed ISV mapping approach. The experiment using COMSOL Multiphysics is to simulate the charging process of a Li-ion battery to observe the concentration 
gradient of different electrodes. Based on these experimental results, the occurrence of Li-plating at different areas and onset times can be successfully identified by employing the developed ISV mapping approach. With the developed approach, a remaining useful life (RUL) prediction strategy considering Li-plating failure mechanism could be further investigated in the future work for battery failure prognostics.

\section{ACKNOWLEDGEMENT}

This research is partially supported by National Science Foundation through Faculty Early Career Development (CAREER) award (CMMI-1351414) and the Award (CMMI-1538508), and by the Department of Transportation through University Transportation Center (UTC) Program.

\section{APPENDIX A. The P2D Model for Li-Ion Battery}

Based on all the principles and kinetics, we can present equations that model the electrochemical dynamics in a Li-ion battery. Integrating Kirchoff's law with Ohm's law, we can obtain the representation of the electric potential the $\Phi_{s}$ in solid phase:

$$
\sigma_{i} \frac{\partial^{2} \Phi_{s}}{\partial x^{2}}=a_{i} F j_{i}, i=p, n
$$

with boundary conditions

$$
-\left.\sigma_{i} \frac{\partial \Phi_{s}}{\partial x}\right|_{x=0, L}=I, \text { and } \Phi_{s, x=0}=0
$$

where $\sigma$ represents the effective electronic conductivity of electrode $i(i=p, n), I$ is the external 
current density, $a$ is the specific surface area of electrode $i, F$ is the Faraday's constant, and $j$ is the wall flux of $\mathrm{Li}^{+}$on the intercalation particle of electrode $i$. At the interface between an electrode and a current collector (i.e. $x=0$ or $L$ in Fig. 1), the first boundary condition in eq. (A.2) is derived because the current density in electrolyte $i_{e}$ is approximated as 0 . The potential in the solid phase of negative electrode is zero as the second boundary condition. The electric potential at two ends of different electrodes can be used to calculate the output voltage as

$$
V=\Phi_{s, x=L}-\Phi_{s, x=0}
$$

The potential in the electrolyte can be calculated as follows:

$$
\frac{\partial \Phi_{e}}{\partial x}=-\frac{i_{e}}{\kappa}+\frac{2 R T}{F}\left(1-t_{+}\right) \times\left(1+\frac{d \ln f_{c / a}}{d \ln c_{e}}\right) \frac{\partial \ln c_{e}}{\partial x}
$$

Since we can only measure differences of potentials, boundary conditions can randomly be set as:

$$
\begin{aligned}
& \Phi_{e, x=L}=0 \\
& \Phi_{e, x=l_{n},-}=\Phi_{e, x=l_{n},+} \text { and } \Phi_{e, x=l_{p},-}=\Phi_{e, x=l_{p},+}
\end{aligned}
$$

where $\kappa$ is the ionic conductivity of the electrolyte, $R$ is the universal gas constant, $T$ is the temperature of the battery, $F$ is the Faraday's constant, $t_{+}$is $L i^{+}$transference number in the electrolyte, and $f_{c / a}$ is the mean molar activity coefficient in electrolyte.

According to the Fick's law, the lithium-ion concentration in the electrolyte is modeled as follows:

$$
\frac{\partial c_{e}}{\partial t}=D_{e f f, i} \frac{\partial^{2} c_{e}}{\partial x^{2}}+a\left(1-t_{+}\right) j_{i}, i=p, n
$$

with the initial condition 


$$
\left.c_{e}\right|_{t=0}=c_{e}^{0}
$$

and the boundary conditions

$$
\left.\frac{\partial c_{e}}{\partial x}\right|_{x=0, L}=0 \text { and }\left.\frac{\partial c_{e}}{\partial x}\right|_{x=l_{i},-}=\left.\frac{\partial c_{e}}{\partial x}\right|_{x=l_{i},+}, i=p, n
$$

where $D_{\text {eff }}$ is the effective diffusion coefficient in electrolyte, $p, n$ represent the positive and negative electrodes, respectively, and $a$ is the specific interfacial area, expressing as

$$
a=\varepsilon_{s}\left(4 \pi R_{p}^{2}\right) /\left[(4 / 3) \pi R_{p}^{3}\right]=\varepsilon_{s}\left(3 / R_{p}\right)
$$

where $\varepsilon_{s}$ represents the volume fraction of the solid electrode material in the porous electrode, $R_{p}$ is the radius of particles in solid phase,

In the solid phase, the transport of the lithium-ion also follows Fick's law similarly, but associates a spherical particle of $R_{p}$ with each particle's location $x$. For each particle at the location $x$, the lithium-ion concentration is described as:

$$
\frac{\partial c_{s}}{\partial t}=\frac{1}{r^{2}} \frac{\partial}{\partial r}\left(D_{s, i} r^{2} \frac{\partial c_{s}}{\partial r}\right), i=p, n
$$

with the initial condition

$$
\left.c_{s}\right|_{t=0}=c_{s}^{0}
$$

and the boundary conditions

$$
\left.\frac{\partial c_{s}}{\partial r}\right|_{r=0}=0 \text { and }\left.\frac{\partial c_{s}}{\partial r}\right|_{r=R_{p}}=-\frac{1}{D_{s}} j_{i}, i=p, n
$$

where $r$ is the radial coordinate of particles in electrodes, $R_{p}$ is the maximum radius of particles, and $D_{s}$ is the diffusion coefficient in solid phase of electrodes 
Relating the net pore-wall molar flux with the divergence of the current, we can obtain the relationship between them at each $\mathrm{x}$ location

$$
\frac{\partial i_{e}}{\partial x}=a F j_{i}, i=p, n
$$

where $\varepsilon_{s}$ is the volume fraction of the solid electrode material in the porous electrode. Besides, the solid phase intercalation overpotential $\eta_{s}$, which determines the rate of the intercalation reaction occurred on the surface of solid particles, is described as

$$
\eta_{s}=\Phi_{s}-\Phi_{e}-U_{r}\left(c_{s, s u f f}\right)-F R_{f} j_{i}
$$

where $U_{r}$ represents the equilibrium potential at the existing surface concentration, $R_{f}$ represents the film resistance of the solid electrolyte interface (SEI).

To associate all the useful variables together and compute the molar flux $j$, the Butler-Volmer equation is employed to build the connection with them. The Butler-Volmer equation is expressed as

$$
j=\frac{i_{0}}{F}\left[\exp \left(\frac{\alpha_{a} F}{R T} \eta_{s}\right)-\exp \left(\frac{-\alpha_{c} F}{R T} \eta_{s}\right)\right]
$$

where $\alpha_{a}$ and $\alpha_{c}$ are the transport coefficients, and $i_{0}$ is the exchange current density, which can be expressed as

$$
i_{0}=r_{e f f} c_{e}^{\alpha_{a}} \times\left(c_{s, \max }-c_{s, \text { surf }}\right)^{\alpha_{a}} \times c_{s, \text { surf }}^{\alpha_{c}}
$$

where $r_{\text {eff }}$ is a constant, and $c_{s, \max }$ is the maximum concentration of lithium-ion in different electrodes depending on specific material properties. Combining all the equations, the output voltage $V$ can be solved by the given input which is the applied current $I$. 


\section{REFERENCES}

[1] Plett, G.L., 2004, "Extended Kalman filtering for battery management systems of LiPBbased HEV battery packs - Part 1. Background," Journal of Power Sources, 134 (2004), 252261.

[2] Zhang, J., Lee, J., 2011, “A review on prognostics and health monitoring of Li-ion battery," Journal of Power Source, 196 (2011), 6007-6014.

[3] Hu, C., Youn, B.D., Chung, J., 2011, “A multiscale framework with extended Kalman filter for lithium-ion battery SOC and capacity estimation," Applied Energy, Vol. 92, 694-704.

[4] Miranda ÁG, Hong CW. Integrated modeling for the cyclic behavior of high power Li-ion batteries under extended operating conditions. Applied Energy. 2013; 111:681-9.

[5] Zheng Y, Ouyang M, Lu L, Li J, Han X, Xu L, et al. Cell state-of-charge inconsistency estimation for LiFePO4 battery pack in hybrid electric vehicles using mean-difference model. Applied Energy. 2013; 111:571-80.

[6] Santhanagopalan, S., Guo, Q., Ramadass, P., and White, R. E., "Review of models for predicting the cycling performance of lithium ion batteries," J. Power Sources, vol. 156, no. 2, pp. 620-628, 2006.

[7] Charkhgard, M., Farrokhi, M., 2010, ' State-of-Charge Estimation for Lithium-Ion Batteries Using Neural Network and EKF," IEEE Transactions on Industrial Electronics, Vol. 57, No. $12,4178-4187$.

[8] Bai, G., Wang, P., Hu, C., \& Pecht, M. (2014). A generic model-free approach for lithiumion battery health management. Applied Energy, 135, 247-260.

[9] Bai, G., Wang, P., \& Hu, C. (2015). A self-cognizant dynamic system approach for 
prognostics and health management. Journal of Power Sources, 278, 163-174.

[10] Sun Y-H, Jou H-L, Wu J-C, Wu K-D. Auxiliary health diagnosis method for lead-acid battery. Applied Energy. 2010; 87:3691-8.

[11] D. Doughty and E. P. Roth, "A General Discussion of Li Ion Battery Safety," Tthe Eletrochemical Society Interface, vol. 21, no.2, summer 2012.

[12] T. F. Fuller, M. Doyle, and J. Newman, "Simulation and Optimation of the Dual Lithium Ion Insertion Cell,” J. Electrochem. Soc., vol. 141, no. 1, pp. 1-10, 1994.

[13] G. Ning and B. N. Popov, "Cycle Life Modeling of Lithium-Ion Batteries," J. Electrochem. Soc., vol. 151, no. 10, pp. A1584-A1591, 2004.

[14] S. Santhanagopalan, Q. Guo, P. Ramadass, and R. E. White, "Review of models for predicting the cycling performance of lithium ion batteries," J. Power Sources, vol.156, pp. $620-628$.

[15] P. Ramadass, B. Haran, P. M. Gomadam, R. E. White, and B. N. Popov, "Development of First Principles Capacity Fade Model for Li-Ion Cells," J. Electrochem. Soc., vol. 151, pp. A196-A203, 2004.

[16] G. Ning, R. E. White, and B. N. Popov, "A generalized cycle life model of rechargeable Li-ion batteries", Electrochimica Acta, vol. 51, pp. 2012-2022, 2006.

[17] L. Cai and R. E. White, “An Efficient Electrochemical-Thermal Model for a Lithium-Ion Cell by Using the Proper Orthogonal Decomposition Method," J. Electrochem. Soc., vol. 157, pp. A1188-A1195, 2010.

[18] M. Guo, G. Sikha, and R. E. White, "Single-Particle Model for a Lithium-Ion Cell: Thermal Behavior," J. Electrochem. Soc., vol.158, pp. A122-A132, 2011.

[19] R. D. Perkins, A. V. Randall, X. Zhang, and G. L. Plett, "Controls oriented reduced order 
modeling of lithium deposition on overcharge," J. Power Sources, vol. 209, pp. 318-325, 2012.

[20] N. Legrand, B. Knosp, P. Desprez, F. Lapicque, and S. Rael, "Physical characterization of the charging process of a Li-ion battery and prediction of Li plating by electrochemical modeling,” J. Power Sources, vol. 245, pp. 208-216, 2014.

[21] N. A. Chaturvedi, R. Klein, J. Christensen, J. Ahmed, and A. Kohic, "Algorithm for Advanced Battery-Management Systems," IEEE Control Systems, vol. 30, pp. 49-68, 2010.

[22] Santhanagopalan, S., Guo, Q., \& White, R. E. (2007). Parameter estimation and model discrimination for a lithium-ion cell. Journal of the Electrochemical Society, 154(3), A198A206.

[23] C. Speltino, D. Domenico, G. Fiengo, A. Stefanopoulou, On the experimental identification of an electrochemical model of a lithium-ion battery: part II, in: The European Control Conference, Budapest, 2009.

[24] A. Schmidt, M. Bitzer, A. Imre, L. Guzzella, Experiment-driven electrochemical modeling and systematic parameterization for a lithium-ion cell, Journal of Power Sources 195 (August) (2010) 5071-5080.

[25] Forman, J. C., Moura, S. J., Stein, J. L., \& Fathy, H. K. (2012). Genetic identification and fisher identifiability analysis of the Doyle-Fuller-Newman model from experimental cycling of a LiFePO4 cell. Journal of Power Sources, 210, 263-275.

[26] Park, M., Zhang, X., Chung, M., Less, G. B., \& Sastry, A. M. (2010). A review of conduction phenomena in Li-ion batteries. Journal of Power Sources, 195(24), 7904-7929.

[27] Ramadesigan, V., Boovaragavan, V., Arabandi, M., Chen, K., Tsukamoto, H., Braatz, R., \& Subramanian, V. (2009). Parameter estimation and capacity fade analysis of lithium-ion 
batteries using first-principles-based efficient reformulated models. ECS Transactions, 19(16), $11-19$. 


\section{List of Tables}

Table 1. Accuracy of the ISV mapping approach 


\section{List of Figures}

Figure 1. The 2D structure of a Li-ion battery

Figure 2: A general neural network model

Figure 3. Procedure of the developed ISV mapping approach

Figure 4. The Li-ion battery 2D geometry in COMSOL

Figure 5. Estimation of diffusion coefficients (DC) in electrodes

(a) Estimation of DC in negative electrode (NE)

(b) Estimation of DC in positive electrode (PE)

Figure 6. Concentration gradient with different combinations of parameters

(a) Concentration gradient with $\mathrm{D}_{\mathrm{n}}=2.013 \mathrm{e}-13$ and $\mathrm{D}_{\mathrm{p}}=1.121 \mathrm{e}-13$

(b) Concentration gradient with $\mathrm{D}_{\mathrm{n}}=2.021 \mathrm{e}-13$ and $\mathrm{D}_{\mathrm{p}}=5.012 \mathrm{e}-14$

(c) Concentration gradient with $\mathrm{D}_{\mathrm{n}}=1.211 \mathrm{e}-14$ and $\mathrm{D}_{\mathrm{p}}=4.986 \mathrm{e}-15$

(d) Concentration gradient with $\mathrm{D}_{\mathrm{n}}=1.122 \mathrm{e}-14$ and $\mathrm{D}_{\mathrm{p}}=5.124 \mathrm{e}-14$ 